\title{
Erratum
}

\section{Canopy-air temperature of crops grown under different irrigation regimes in a temperate humid climate}

\author{
H. E. Jensen, H. Svendsen, S. E. Jensen, and V. O. Mogensen \\ Department of Soil and Water and Plant Nutrition, The Royal Veterinary and Agricultural University, Thorvaldsensvej 40 , \\ DK-1871 Frederiksberg C, Copenhagen, Denmark
}

Irrigation Science, Volume 11, Number 3, pp 181-188

Table 1. Linear regression parameters calculated for $\left(T_{c}-T_{a}\right)$ versus $\left(e_{a}^{*}-\mathrm{e}_{\mathrm{a}}\right)$ for rape and barley. $N$ is number of observations, $a$ and $b$ are intercept and slope, respectively, $s_{a}$ and $s_{b}$ are standard deviation of intercept and slope, respectively, while $R$ is regression coefficient

\begin{tabular}{llrllllll}
\hline Crop & \multirow{2}{*}{ Period } & \multicolumn{6}{c}{ Linear regression parameters } \\
\cline { 3 - 8 } & & $a$ & $s_{a}$ & $b$ & $s_{b}$ & $R^{2}$ & $N$ \\
\hline Rape & Total & 4.5 & 0.8 & -2.7 & 0.6 & 0.47 & 28 \\
Rape & Preflowering & 6.7 & 1.7 & -4.6 & 1.3 & 0.62 & 10 \\
Rape & Postflowering & 4.5 & 1.0 & -2.6 & 0.7 & 0.49 & 18 \\
Barley & Total & 4.2 & 0.4 & -2.6 & 0.3 & 0.61 & 43 \\
Barley & Preheading & 3.1 & 0.3 & -1.9 & 0.2 & 0.68 & 38 \\
Barley & Postheading & 15.9 & 1.7 & -14.1 & 1.8 & 0.96 & 5 \\
\hline
\end{tabular}

Table 2. Linear regression parameters calculated for $\left(T_{c}-T_{a}\right)$ versus $\left(e_{a}^{*}-e_{a}\right)$ for rape and barley at various levels of global radiation $\left(S_{i}\right)$ and wind speed $(\mu) . N$ is number of observations, $a$ and $b$ are intercept and slope, respectively, $s_{a}$ and $s_{b}$ are standard deviation of intercept and slope, respectively, while $R$ is regression coefficient

\begin{tabular}{lllllllrr}
\hline$S_{i}\left(\mathrm{~W} \mathrm{~m}^{-2}\right)$ & $\mu\left(\mathrm{m} \mathrm{s}^{-1}\right)$ & \multicolumn{8}{c}{ Regression parameters } \\
\cline { 3 - 8 } & & $a$ & $s_{a}$ & $b$ & $s_{b}$ & $R^{2}$ & $N$ \\
\hline Rape & & & & & & & \\
$600-800$ & all obs. & 3.4 & 1.0 & -2.2 & 0.7 & 0.38 & 17 \\
$800-1000$ & all obs. & 2.7 & 0.7 & -1.3 & 0.5 & 0.54 & 9 \\
all obs. & $2-4$ & 4.6 & 0.9 & -2.6 & 0.6 & 0.50 & 21 \\
all obs. & $4-6$ & 5.8 & 2.3 & -4.2 & 1.7 & 0.61 & 6 \\
Barley & & & & & & & \\
$600-800$ & all obs. & 2.9 & 0.4 & -1.7 & 0.3 & 0.63 & 26 \\
$800-1000$ & all obs. & 3.6 & 0.4 & -2.1 & 0.3 & 0.81 & 14 \\
all obs. & $2-4$ & 4.8 & 0.6 & -2.8 & 0.4 & 0.67 & 23 \\
all obs. & $4-6$ & 2.9 & 0.6 & -1.7 & 0.4 & 0.53 & 18 \\
\hline
\end{tabular}

Table 3. Linear regression parameters calculated for $\left(T_{c}-T_{a}\right)$ versus $\left(e_{a}^{*}-e_{a}\right)$ for wheat and perennial rye grass. $N$ is number of observations, $a$ and $b$ are intercept and slope, respectively, $s_{a}$ and $s_{b}$ are standard deviation of intercept and slope, respectively, while $R$ is regression coefficient

\begin{tabular}{lllllllll}
\hline Crop & Period & \multicolumn{6}{c}{ Linear regression parameters } \\
\cline { 3 - 8 } & & $a$ & $s_{a}$ & $b$ & $s_{b}$ & $R^{2}$ & $N$ \\
\hline Wheat & Total & 2.9 & 0.8 & -3.1 & 1.5 & 0.09 & 47 \\
Wheat & Preheading & 8.5 & 2.6 & -12.0 & 5.3 & 0.35 & 11 \\
Wheat & Postheading & 1.9 & 0.8 & -1.9 & 1.4 & 0.05 & 36 \\
Ryegrass & Total & 2.2 & 0.7 & -0.7 & 1.4 & 0.01 & 72 \\
\hline
\end{tabular}

Table 4. Linear regression parameters calculated for $\left(T_{c}-T_{a}\right)$ versus $\left(\mathrm{e}_{\mathrm{a}}^{*}-\mathrm{e}_{\mathrm{a}}\right)$ at various levels of global radiation $\left(\mathrm{S}_{\mathrm{i}}\right)$ and wind speed $(\mu)$, respectively, for wheat. $N$ is number of observations, $a$ and $b$ are intercept and slope, respectively, $s_{a}$ and $s_{\mathrm{b}}$ are standard deviation of intercept and slope, respectively, while $R$ is regression coefficient

\begin{tabular}{llllllllr}
\hline$S_{i}\left(\mathrm{~W} \mathrm{~m}^{-2}\right)$ & $\mu\left(\mathrm{m} \mathrm{s}^{-1}\right)$ & \multicolumn{6}{c}{ Linear regression parameters } \\
\cline { 3 - 8 } & & $a$ & $s_{a}$ & $b$ & $s_{b}$ & $R^{2}$ & $N$ \\
\hline $200-400$ & all obs. & 3.2 & 2.8 & -8.0 & 7.5 & 0.22 & 6 \\
$400-600$ & all obs. & 3.2 & 1.5 & -4.9 & 2.7 & 0.23 & 13 \\
$600-800$ & all obs. & 3.9 & 1.2 & -4.1 & 2.1 & 0.20 & 18 \\
$800-1000$ & all obs. & 7.8 & 0.9 & -9.9 & 1.6 & 0.82 & 10 \\
all obs. & $0-2$ & 0.6 & 1.9 & 2.3 & 4.1 & 0.05 & 8 \\
all obs. & $2-4$ & 3.9 & 1.4 & -4.3 & 2.6 & 0.14 & 19 \\
all obs. & $4-6$ & 1.9 & 1.4 & -1.5 & 2.5 & 0.02 & 18 \\
\hline
\end{tabular}


Table 5. Linear regression parameters calculated for $\left(T_{c}-T_{a}\right)$ versus $\left(e_{a}^{*}-e_{a}\right)$ at various levels of global radiation $\left(S_{i}\right)$ and wind speed $(\mu)$, respectively, for ryegrass. $N$ is number of observations, $a$ and $b$ are intercept and slope, respectively, $s_{a}$ and $s_{b}$ are standard deviation of intercept and slope, respectively, while $R$ is regression coefficient

\begin{tabular}{llrlllllr}
\hline$S_{i}\left(\mathrm{~W} \mathrm{~m}^{-2}\right)$ & $\mu\left(\mathrm{m} \mathrm{s}^{-1}\right)$ & \multicolumn{6}{c}{ Linear regression parameters } \\
\cline { 3 - 8 } & & \multicolumn{1}{c}{$a$} & $s_{a}$ & $b$ & & $s_{b}$ & $R^{2}$ & $N$ \\
\hline $200-400$ & all obs. & 2.2 & 0.9 & -4.2 & 2.3 & 0.18 & 17 \\
$400-600$ & all obs. & 4.6 & 1.2 & -6.2 & 2.3 & 0.28 & 20 \\
$600-800$ & all obs. & 4.1 & 0.9 & -2.6 & 1.6 & 0.08 & 32 \\
$800-1000$ & all obs. & 11.3 & 1.3 & -14.9 & 2.3 & 0.85 & 9 \\
all obs. & $0-2$ & 3.2 & 1.3 & -1.7 & 2.5 & 0.03 & 18 \\
all obs. & $2-4$ & 5.7 & 1.2 & -6.4 & 2.2 & 0.23 & 31 \\
all obs. & $4-6$ & 0.2 & 0.9 & 2.3 & 1.7 & 0.05 & 36 \\
all obs. & $6-8$ & -1.8 & 2.0 & 4.6 & 3.0 & 0.53 & 4 \\
\hline
\end{tabular}

Table 6. Apparent values of aerodynamic resistance $\left(r_{a}\right)$ and crop resistance $\left(r_{c p}\right)$ calculated from estimated lower base lines using average values of net radiation $\left(R_{n}\right)$ and slope of the saturated vapor pressure temperature relation ( 4 ) at two levels of global radiation $\left(S_{\mathrm{i}}\right)$

\begin{tabular}{llllll}
\hline Crop & $\begin{array}{l}S_{i} \\
\left(\mathrm{~W} \mathrm{~m} \mathrm{~m}^{-2}\right)\end{array}$ & $\begin{array}{l}R_{n} \\
\left(\mathrm{~W} \mathrm{~m}^{-2}\right)\end{array}$ & $\begin{array}{l}\Delta \\
\left(\mathrm{Pa}^{\circ} \mathrm{C}^{-1}\right)\end{array}$ & $\begin{array}{l}r_{\mathrm{a}} \\
\left(\mathrm{s} \mathrm{m}^{-1}\right)\end{array}$ & $\begin{array}{l}r_{c} \\
\left(\mathrm{~s} \mathrm{~m}^{-1}\right)\end{array}$ \\
\hline Rape & $600-800$ & 514 & 163 & 13 & 44 \\
& $800-1000$ & 594 & 167 & 7 & 59 \\
Barley & $600-800$ & 516 & 166 & 10 & 53 \\
& $800-1000$ & 608 & 166 & 12 & 42 \\
\hline
\end{tabular}

